Seventh International Course on Dengue: A Challenge for the Third Millennium Local: Instituto Pedro Kourí, Havana, Cuba Data: 13 a 24 de agosto de 2001 Informações: Prof. María G. Guzmán, Instituto Pedro Kourí, Autopista Novia del Mediodía, Km 6, P. O. Box 601, Mnao. 13, Ciudad Havana, Cuba Tel: 53-7-220450, 53-7-220633; Fax: 53-7-246051 lupe@ipk.sld.cu http://www.sld.cu/instituciones/ipk/ eventoipk/cdengue.htm

XVth International Congress

of the European Society for Philosophy of Medicine and Healthcare

Local: Lisboa, Portugal

Data: 16 a 18 de agosto de 2001

Tema: "Wisdom in Healthcare", including kairos in clinical decision-making, philosophy and clinical epidemiology, evidence-based medicine, care ethics, the wise healthcare professional, the wise patient, the wise healthcare policymaker

Informações: Prof. Dr. Henk ten Have, secretariat of the European Society for Philosophy of Medicine and Healthcare, Dept. of Ethics, Philosophy and History of Medicine, Faculty of Medical Sciences, Catholic University of Nijmegen, P.O. Box 9101, 6500 HB Nijmegen, The Netherlands Fax: (31) 24-3504254

XXXIV International Congress of Physiological Sciences, From Molecule to Malady Local: Christchurch, Nova Zelândia Data: 26 a 31 de agosto de 2001 Informações: Peter S. Davie, Associate Professor. Comparative Physiology \& Anatomy Section, Massey University, Private Bag, 11222 , Palmerston North, New Zealand. Tel: +64-6-3504472; Fax: +64-6-3505674 http://www.iups2001.org.nz

17th International Congress of Nutrition: Modern Aspects of Nutrition - Present Knowledge and Future Perspectives Local: Viena, Áustria

Data: 27 a 31 de agosto de 2001

Informações: Ibrahim Elmadfa, Institute of Nutritional Sciences, University of Vienna. Altharstr 14 (Pharaziezentrum), A-1090 Vienna Tel: +43 1 313368213; Fax: +43131336773 ibrahim.elmadfa@univie.ac.at http://www.univie.ac.at/iuns2001
Third International Congress of Vector Ecology Local: Barcelona, Espanha

Data: 16 a 21 de setembro de 2001

committee@sove2001.org

http://www.sove2001.org

\section{Congress of the International Association} for Suicide Prevention

Local: Chenna, Índia

Data: 22 a 26 de setembro de 2001

Informações: Congress Secretariat Tel: +91 44 4470312; Fax: +91 444473611 info@iasp2001.org http://www.iasp2001.org

Injury Prevention 2001. 4th National Farm Injury Prevention Conference and the 5 th Australian Injury Prevention \& Control Conference Local: Deakin University, Warrnambool, Victoria, Austrália

Data: 25 a 28 de setembro de 2001 Tema: Farm safety; Indigenous safety; Personal safety; Rural occupational health and safety; Safe communities; Safe sport; Travel safety Informações: Injury Prevention 2001 Conference Secretariat, Glenormiston College, PMB 6200, TERANG Victoria 3264 Australia Tel: +61 3 55578200; Fax: +61 355578200 injury-prevention@unimelb.edu.au

Il Congresso Brasileiro de Biossegurança e II Simpósio Latino-Americano de Produtos Transgênicos

Local: Salvador, Bahia, Brasil

Data: 26 a 28 de setembro de 2001

http://www.anbio.org.br

$X V$ Congresso Latino-Americano de Parasitologia, XVII Congresso Brasileiro de Parasitologia e I Congresso da Sociedade Paulista de Parasitologia

Local: Centro de Convenções Rebouças, São Paulo, São Paulo, Brasil

Data: 7 a 11 de outubro de 2001

Tema: Parasitologia no Novo Milênio

Tel: (11) 31410707

Fax: (11) 2846629

planetevents@planetevents.com

33rd Meeting of the Internatinal Society of Pediatric Oncology

Local: Queensland, Austrália

Data: 10 a 13 de outubro de 2001

Informações: SIOP 2001 Secretariat, P. O. Box 1280, 11/97 Castlemaine St., Milton, QLD 4064, Austrália Tel: 61 (0) 738585580 ; Fax: 61 (0) 738585510 siop2001@im.com.au 
XIX Reunión Científica de la Sociedad Española de Epidemiología

Local: Murcia, Espanha

Data: 17 a 19 de outubro de 2001

Tema: Ética, medio ambiente y comunicación: punto de encuentro de la epidemiologia en el siglo XXI Informações: Alquibla Congresos,

Pintor Villacis 4 - 30003 - Murcia, España

Tel: 9682250 20; Fax: 968225154

info@alquibla.com

see2001@carm.es

http://www.um.es/siu/congre/see-murcia2001

2nd International Symposium on Obesity and Hypertension

Local: Berlim, Alemanha

Data: 25 a 27 de outubro de 2001

Informações: CTW - Congress Organisation

Thomas Wiese Gmbh, Goßlenstr. 30,

D-12161 Berlin, Germany

Tel: (++49 30) 85996217; Fax: (++49 30) 85079826

alexandra.volk@ctw-congress.de

http://www.ctw-congress.de

XXXIII Congresso da Associação Latino-Americana de Sociologia

Local: Universidad de San Carlos,

Guatemala, Guatemala

Data: 29 de outubro a 2 de novembro de 2001

Tema: América Latina: entre la globalización del subdesarollo y la emergencia de nuevas alternativas. Los urgentes desafios del pensamiento crítico latinoamericano

\section{2nd World Conference on Lung Health}

Local: Paris, França

Data: 1o a 4 de novembro de 2001

Informações: International Union Against

Tuberculosis and Lung Diseases, 68, Boulevard

Saint-Michel - 75006 Paris, France

Tel: (+33) 1443203 60; Fax: (+33) 143299087

union@iuatld.org

http://www.iuatld.org

50th Annual Meeting of the American Society of Tropical Medicine and Hygiene

Local: Hilton Atlanta Hotel And Towers, Atlanta,

Gerogia, Estados Unidos

Data: 11 a 15 de novembro de 2001

Informações: American Society of Tropical

Medicine and Hygiene

Tel: 847-480-9592

astmh@astmh.org

http://www.astmh.org
8 Simpósio Internacional sobre Esquistossomose Local: Recife Lucsim Palace Hotel, Recife, Brasil Data: 2 a 5 de dezembro de 2001

Tema: Epidemiologia e controle; Aspectos sociais e antropologia; Biologia, genética e imunologia de moluscos transmissores; Relações hospedeiroparasito; Biologia molecular do parasito; Imunidade protetora e desenvolvimento de vacinas; Patogênese e imunopatologia; Avanços recentes em diagnósticos e tratamento; Ultra-sonografia como instrumento de diagnóstico; Hipertensão portal na esquistossomose Informações: CEJEM - Secretaria Executiva.

Rua Gal. Americano Freire, 394, sala 404, Boa Viagem, Recife, PE 51021-120, Brasil Tel: (81) 34664723/34665551/34658504 Fax: (81) 33255015 cejem@elogica.com.br http://www.cpqam.fiocruz.br/eventos/ symposium.html

\section{8ㅇ Congresso da Sociedade Brasileira} de Medicina Tropical

Local: Foz do Iguaçu, Paraná, Brasil

Data: 24 a 28 de fevereiro de 2002

Informações: Ekipe de Eventos, Congressos e Feiras. Av. Sete de Setembro, 4857, Sobreloja, Curitiba, PR 80240-000, Brasil

Tel/Fax: (41) 342-1247

ekipedeeventos@avalon.sul.com.br

\section{Fourth International Congress}

on Vegetarian Nutrition

Local: Loma Linda, California, Estados Unidos

Data: 17 a 22 de março de 2002

Informações: Department of Nutrition, School

of Public Health, Loma Linda University,

Loma Linda, CA 92350

ICVN@sph.llu.edu

6th World Conference on Injury

Prevention and Control

Local: Montreal, Quebec, Canadá

Data: 12 a 15 de maio de 2002

Informações: 511, place d'Armes, \#600,

Montreal QC H2Y sW7, Canada

Tel: 514848 1133; Fax: 5142886469

trauma@coplanor.qc.ca

http://www.trauma2002.com

\section{Cruzando Fronteras en América Latina.}

Tercer Congreso Europeo de Latinoamericanistas Local: Amsterdam, Holanda

Data: 3 a 6 de julho de 2002

Informações: CEISAL 2002, c/o CEDLA,

Keizersgracht 395-397, 1016 EK Amsterdam, Holanda Fax: (+31 20) 6255127

ceisal02@cedla.uva.nl

http://www.cedla.uva.nl/ceisal-2002 